\title{
Research on Passenger Terminal Layout Planning of Metropolitan Coordination Region
}

\author{
Huimin Zheng ${ }^{1, a}$ and Xuan Chen ${ }^{1, b}$ \\ ${ }^{1}$ Qingdao Binhai University, West Jialingjiang Road, Development Zone of Qingdao, Shandong, \\ China \\ azhmhappy@126.com, ${ }^{\mathrm{b}}$ chenxuanjt@163.com
}

Keywords: MCR, Combination terminal, Covering model

\begin{abstract}
With the rapid social and economic development and urbanization process speeding up, at present planning and construction of highway passenger terminal in Metropolitan Coordination Region (MCR) [1] has lagged behind and cannot meet the development needs of economic integration. Therefore, the layout of highway passenger terminal planning in MCR is very important. Single and multiple terminal location models are created based on improved differential method and covering model respectively. Then, the feasible solution is obtained by using the heuristic algorithm. Due to the comprehensive consideration of the method, it will play an important role in the location of the passenger terminal in the metropolitan area.
\end{abstract}

\section{Introduction}

The MCR is directly derived from the work of Jan Gottman, a French geologist, on the word "Megalopolis" [2], which is mentioned in the metropolitan area: the urbanization of the northeast coast. The convenient and efficient transportation is the basic premise of economic development. The MCR is a complex composed of the core city and several related peripheral cities [3]. Compared to a single city, a proper MCR can achieve economies of scale and maximize the efficiency of regional resource.

Competition in the future is the MCR than a city. Passenger terminals are important support for the economy in MCR, research on layout planning methods should be paid more attention to [4-6]. The lack of scientific and systematic layout planning will inevitably lead to the unreasonable distribution and decentralized management of the MCR Terminal facilities, which will result in duplication, excessive construction and investment waste and efficacy of the attenuation [7].

\section{Basic Concepts}

Passenger Terminal with clear hierarchy, perfect structure and clear division can provide a good basis for the determination of investment, management and construction scale. The efficiency and level of service of the whole transport system can be improved through cooperation among different levels of passenger terminal. The combined terminals can be set up according to the interdependent relation of cities. They can combine the geographical, cultural, transportation and other conditions [5], give full play to the efficiency of the use of transport terminals which can avoid duplication, reduce waste of resources and the excessive competition in their operations.

According to the above idea, the layout planning of MCR passenger transport can be divide three levels: National terminals, Provincial terminals and the MCR terminals. They can also form combination terminals.

\section{Models}

Passenger terminal layout planning is a very complicated process. In order to get more reasonable results, we should follow the point of view of engineering system. When need to add a single terminal in the area, an improved differential method for hierarchical division can be build with models for centroid and differentiation method[6]. When need to determine multiple terminals, the 
heuristic algorithm and the maximum coverage model are used to solve the location of passenger terminal [7].

The objective function $\mathrm{T}$ is the total cost of the passenger flow in the area, which contains the cost of occurrence and attraction point to reach the station.

$$
T=\sum_{j=1}^{n} C_{j} W_{j}\left[\left(x-x_{i}\right)^{2}+\left(y-y_{i}\right)^{2}\right]^{1 / 2}
$$

If the lowest total cost $\mathrm{T}$ will be calculated, the partial differential of $\mathrm{T}$ to $\mathrm{x}$ and $\mathrm{T}$ to $\mathrm{y}$ should be set to zero. The new extreme points are obtained, Solution formulas are:

$$
\left\{\begin{array}{c}
x=\frac{\sum_{j=1}^{n} C_{j} W_{j} x_{j}\left[\left(x-x_{i}\right)^{2}+\left(y-y_{i}\right)^{2}\right]^{1 / 2}}{\sum_{j=1}^{n} C_{j} W_{j}\left[\left(x-x_{i}\right)^{2}+\left(y-y_{i}\right)^{2}\right]^{1 / 2}} \\
y=\frac{\sum_{j=1}^{n} C_{j} W_{j} y_{j}\left[\left(x-x_{j}\right)^{2}+\left(y-y_{j}\right)^{2}\right]^{1 / 2}}{\sum_{j=1}^{n} C_{j} W_{j}\left[\left(x-x_{j}\right)^{2}+\left(y-y_{j}\right)^{2}\right]^{1 / 2}}
\end{array}\right.
$$

In the table: $\mathrm{n}$ is external passenger flow generating point; $W_{j}(j=1,2, \cdots, n)$ is the occurrence of each point; $\left(x_{i}, y_{j}\right)_{\text {is coordinate; }}(x, y)$ is planning station coordinate; ${ }^{C_{j}}$ is the cost of transportation system.

\section{Applications}

As shown in Fig. 1, assuming the following 11 points are large demand points (In order to simplify the calculation, assuming the large demand point, is the initial position of the passenger station). The travel time between demand points and the terminals has been marked (min). Due to objective reasons, demand point 10 is not suitable for the construction of passenger stations, other points can be set. In order to improve the level of service, the time required for each demand point to reach the passenger terminal station is not more than $45 \mathrm{~min}$. In order to meet the needs, at least how many passenger transport terminals need to be built?

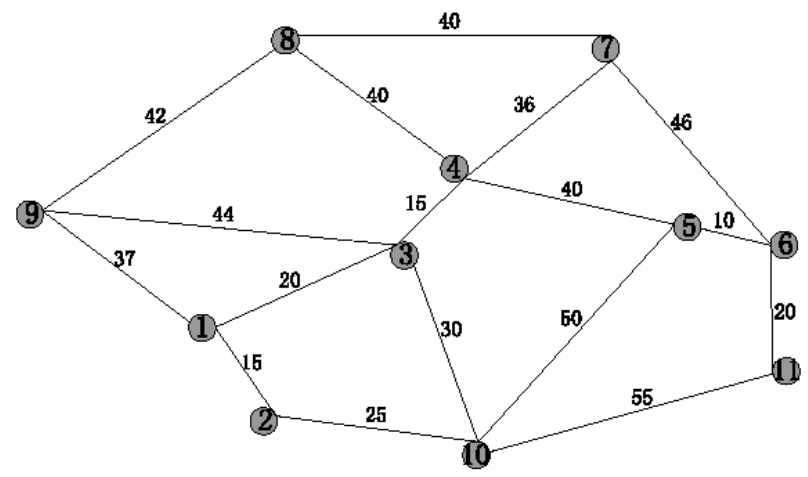

Figure 1. Passenger transport demand and time distribution of the MCR

1) The heuristic algorithm is used to solve the problem

The solution procedure is as follows:

First, identify the range of requirement and service that each point provides, and then the points are marked as A (j), B (i), as shown in Table 1. 
Table 1 Initial node service range

\begin{tabular}{|c|c|c|}
\hline Points & $A(j)$ & $B(i)$ \\
\hline 1 & $1,2,3,9$ & $1,2,3,9$ \\
\hline 2 & $1,2,10$ & $1,2,10$ \\
\hline 3 & $1,3,4,9,10$ & $1,3,4,9,10$ \\
\hline 4 & $3,4,5,8$, & $3,4,5,8$ \\
\hline 5 & $4,5,6$ & $4,5,6$ \\
\hline 6 & $5,6,11$ & $5,6,11$ \\
\hline 7 & $4,7,8$ & $(4,7,8)$ \\
\hline 8 & $4,7,8,9$ & $4,7,8,9$ \\
\hline 9 & $1,3,8,9$ & $1,3,8,9$ \\
\hline 10 & $2,3,10$ & $(2,3)$ \\
\hline 11 & 6,11 & $(6,11)$ \\
\hline
\end{tabular}

Using the heuristic algorithm, a set of feasible solutions are obtained: $(6,8,2,3),(6,8,2,4),(6$, $8,2,9),(6,8,1,2),(6,8,1,3)$, which is as an initial solution. In order to get the best solution, it is necessary to optimize the initial solution.

2) Using the method of correlation analysis to optimize the scheme

Five feasible solutions are obtained in the model solution: $(6,8,2,3),(6,8,2,4)(6,8,2,9),(6,8$, $1,2),(6,8,1,3)$. In the feasible solution, two points need to be selected from $(1,2,3,4,9)$. Assuming the 5 points of the various indicators and the weight value of the index is shown in Table 2.

Table 2 Alternative point table for passenger terminal

\begin{tabular}{|c|c|c|c|c|c|c|}
\hline Optimization index & \multirow{2}{*}{ weight } & \multicolumn{5}{|c|}{ alternative point } \\
\cline { 4 - 7 } & & 1 & 2 & 3 & 4 & 9 \\
\hline Covering area(million $\mathrm{m}^{2}$ ) & 0.11 & 0.65 & 0.60 & 0.80 & 0.70 & 0.65 \\
\hline Maximum assembling(people) & 0.19 & 1000 & 1200 & 2000 & 1500 & 1300 \\
\hline Traffic demand(ten thousand people) & 0.18 & 0.8 & 1.1 & 1.2 & 1 & 1.3 \\
\hline Land condition(ten thousand $\mathrm{m}^{2}$ ) & 0.08 & 4 & 6 & 7.2 & 5 & 3.6 \\
\hline Transfer convenience & 0.13 & 0.6 & 0.8 & 0.76 & 0.8 & 0.7 \\
\hline $\begin{array}{c}\text { Expected passenger flow(Ten thousand } \\
\text { people) }\end{array}$ & \multirow{2}{*}{0.17} & 1.2 & 1 & 2 & 1.3 & 1.4 \\
\hline Coordination with the overall planning & 0.10 & 0.78 & 0.92 & 0.89 & 0.75 & 0.8 \\
\hline Environmental impact & 0.04 & 0.8 & 0.7 & 0.6 & 0.72 & 0.75 \\
\hline
\end{tabular}

Solution methods are as follows:

Step 1: the supplementary matrix of the index matrix is

$$
E=\left[\begin{array}{cccc}
f_{10} & f_{11} & \cdots & f_{1 m} \\
f_{20} & f_{21} & \cdots & f_{2 m} \\
\cdots & \cdots & \cdots & \cdots \\
f_{n 0} & f_{n 1} & \cdots & f_{n m}
\end{array}\right]=\left[\begin{array}{cccccc}
80 & 65 & 60 & 80 & 70 & 65 \\
2000 & 1000 & 1200 & 2000 & 1500 & 1300 \\
1.3 & 0.8 & 1.1 & 1.2 & 1 & 1.3 \\
7.2 & 4 & 6 & 7.2 & 5 & 3.6 \\
0.8 & 0.6 & 0.8 & 0.76 & 0.8 & 0.7 \\
2 & 1.2 & 1 & 2 & 1.3 & 1.4 \\
0.92 & 0.78 & 0.92 & 0.89 & 0.75 & 0.8 \\
0.25 & 0.5 & 0.3 & 0.4 & 0.36 & 0.25
\end{array}\right]
$$


Step 2: after the addition of a matrix of non-dimensional treatment to get the matrix

$$
F^{\prime}=\left[\begin{array}{lllll}
1 & f_{11}^{\prime} & f_{12}^{\prime} & \cdots & f_{1 m}^{\prime} \\
1 & f_{21}^{\prime} & f_{22}^{\prime} & \cdots & f_{2 m}^{\prime} \\
\cdots & & & & \\
1 & f_{8,1}^{\prime} & f_{8,2}^{\prime} & \cdots & f_{8, m}^{\prime}
\end{array}\right]=\left[\begin{array}{cccccc}
1 & 0.81 & 0.75 & 1 & 0.88 & 0.81 \\
1 & 0.5 & 0.6 & 1 & 0.75 & 0.65 \\
1 & 0.62 & 0.85 & 0.92 & 0.77 & 1 \\
1 & 0.56 & 0.83 & 1 & 0.69 & 0.5 \\
1 & 0.75 & 1 & 0.95 & 1 & 0.88 \\
1 & 0.6 & 0.5 & 1 & 0.65 & 0.7 \\
1 & 0.85 & 1 & 0.97 & 0.82 & 0.87 \\
1 & 2 & 1.2 & 1.6 & 1.44 & 1
\end{array}\right]
$$

Step 3: calculate the correlation coefficient between the corresponding indicators

$$
D=\left[\begin{array}{cccc}
\eta_{11} & \eta_{12} & \cdots & \eta_{1,5} \\
\eta_{21} & \eta_{22} & \cdots & \eta_{2,5} \\
\cdots & \cdots & \cdots & \cdots \\
\eta_{81} & \eta_{82} & \cdots & \eta_{8,5}
\end{array}\right]=\left[\begin{array}{ccccc}
0.73 & 0.67 & 1 & 0.8 & 0.73 \\
0.5 & 0.56 & 1 & 0.67 & 0.59 \\
0.57 & 0.76 & 0.87 & 0.68 & 1 \\
0.53 & 0.75 & 1 & 0.63 & 0.5 \\
0.67 & 1 & 0.91 & 1 & 0.8 \\
0.56 & 0.5 & 1 & 0.59 & 0.63 \\
0.77 & 1 & 0.94 & 0.73 & 0.79 \\
0.33 & 0.71 & 0.45 & 0.53 & 1
\end{array}\right]
$$

Step 4: calculate the degree of correlation

Weight of each index:

$$
\bar{W}=\left(w_{1}, w_{2}, \cdots, w_{8}\right)=(0.11,0.19,0.18,0.08,0.13,0.17,0.1,0.04)
$$

$\mathrm{D}$ is the correlation coefficient matrix, then vector

$$
\left.\bar{R}=\bar{W} \bullet D=(0.59,0.72,0.94,0.71,0.74) \quad r_{j}^{*}=\max _{j} x r_{j}\right\}=\left\{r_{3}\right\}
$$

According to the optimal degree of relevance, the node 3 is selected. So $(6,8,2,3),(6,8,1,3)$ were selected. The correlation degree of node 2 is greater than 1 . Finally the optimization program $(6,8,2,3)$ is get.

Considering the influence of node importance on the terminal level, node 2,6,8 is located in the core city, so they are national terminals. Similarly, node 3 is the MCR terminals. If the surrounding has a small station, they can also form combination terminals.

\section{Conclusions}

The integration of regional economy puts forward higher requirements to the transportation conditions of the MCR. Single and multiple terminal location models are created based on improved differential method and covering model respectively. It should be noted that when the feasible solution of the model is not unique, the most scientific and practical solution in many feasible schemes need to be seek out. Correlation analysis method of grey theory is used to optimize the planning scheme.

As a result of regional economic integration, management system of the MCR has not yet formed. How to overcome the limitation of administrative division; coordinate the various departments in the MCR, make the plan truly, further research is needed. 


\section{References}

[1] Gottmann J. Megalopolis, or the Urbanization of the Northeastern Seaboard [J] Economic Geography, 1957, 33 (7):31-40.

[2] QinXu. From the experience of the development of the world city circle to talk about the construction of China's Metropolitan Are [J] Journal of Nanjing University of Technology, 2002(3):56-63. (In Chinese)

[3] Jun Zou, Xuefeng Wang et al. Urban planning. [M], Beijing: China Building Industry Press, 2005.3-6. (In Chinese)

[4] Mengyu Cao. The study on Yangtze River Delta region highway passenger transport linkage [D]. Shanghai: Shanghai Normal University, 2013. (In Chinese)

[5] Wenjuan An. Analysis and prediction of passenger demand structure of regional transport corridor [D]. Beijing: Beijing Jiaotong University, 2011. (In Chinese)

[6] Chunfu Shao. Traffic planning [M]. Beijing: China Railway Publishing House, 2015.12. (In Chinese)

[7] D. Shanghai: Shanghai Normal University, 2013The Ministry of communications of the People's Republic of China. The layout of the national highway transportation hub planning and delivery, 2007. (In Chinese)

[8] Dr. Jena-Paul Rodrigue. Transportation Terminals. The Geography of Transport Systems, 2001.

[9] Bixia Fan. Application of set covering model in logistics center location, decision making \& Information [J]. 2008, (9):49-50. (In Chinese)

[10]Dong Du, Qinghua Pang. Modern comprehensive evaluation methods and case study [M] Beijing: Tsinghua University press, 2005. (In Chinese) 